\title{
Attending to simple auditory and visual signals
}

\author{
ROBERT M. MULLIGAN and MARILYN L. SHAW \\ Rutgers-The State University, New Brunswick, New Jersey 08903
}

\begin{abstract}
Is the quality of information obtained from simple auditory and visual signals diminished when both modalities must be attended to simultaneously? This question was investigated in an experiment in which subjects made forced-choice judgments of the location of simple light and tone signals presented in focused- and divided-attention conditions. The data are compared with the predictions of a model that describes the largest performance decrement to be expected in the divided-attention condition on the basis of nonattentional factors. The results of this comparison suggest that the difference in performance between focused- and dividedattention conditions is attributable solely to the increased opportunity to confuse signal with noise as the number of modalities is increased. Thus, there appears to be no evidence that dividing attention between modalities affects the quality of the stimulus representations of individual light and tone signals.
\end{abstract}

The experiment described in this paper examines the consequences of attending simultaneously to several sources of information. The specific situation investigated involves attending to multimodal inputsimple auditory and visual stimuli. It has often been observed that as the number of information sources or channels (e.g., spatial locations, sensory modalities, spatial frequency channels, etc.) requiring attention is increased, performance for individual sources is diminished. Similar findings have been observed with several tasks, including full report, detection, and forced choice (for reviews, see Egeth, 1977; Gardner, 1973; Schneider \& Shiffrin, 1977; Shaw, Note 1). This common result would seem to indicate the operation of a capacity limitation resulting in a decrease in the amount of attention allocated to individual items. It has been pointed out, however, in several discussions of attention and capacity limitation (Eriksen \& Spencer, 1972; Gardner, 1973; Kinchla, 1969; Shiffrin \& Gardner, 1972), that such a performance decrement can be accounted for simply by the fact that the decision processes are more complicated when the number of sources requiring attention is increased. In particular, a drop in accuracy is predicted due to the purely statistical increase in the probability of confusing signal with noise as the number of signal sources is increased.

The question that must be addressed, then, is the following: Does the performance decrement observed upon increasing the number of to-be-attended to sources reflect a diminution in the quality of information obtained from each source, or is it merely a statistical consequence of increasing the subject's

This research was supported by NSF Grant BNS 79-24885 to the second author. Some of the data were presented at the 21 st annual meeting of the Psychonomic Society, St. Louis, November 1980. opportunities for making errors? Changes in performance attributable to these two types of factors will be referred to as "attentional" and "decisonal" effects, respectively. There has been some disagreement regarding this question in the literature on dividing attention between modalities. For example, Eijkman and Vendrik (1965) and Shiffrin and Grantham (1974) presented results which were interpreted as showing no divided attention deficit. In contrast to these results, Massaro and Kahn (1973) and Massaro and Warner (1977) observed a significant decrement in performance in tasks requiring simultaneous attention to two modalities when compared with unimodal conditions, and have interpreted these findings in terms of attentional deficits. Fidell (1970) considered both attentional ("uncertainty reduction") and decisional ("noise reduction") explanations for an observed difference in performance between focused and divided attention conditions, but his analysis did not discriminate between the two explanations. There are caveats to each of these results such that none of them provide conclusive evidence either for or against an attentional effect.

In the present paper, we will attempt to distinguish attentional from decision-making components in a multimodal attention task, making use of a theoretical framework developed recently by one of us (Shaw, 1980). The model to be described has been used successfully in previous studies examining the effects of set size on performance in a visual search task (Shaw \& Mulligan, Note 2). Before describing the model, it will be useful to outline the paradigm in which the multimodal attention effects were investigated. In the paradigm, focused and divided attention with respect to modality were contrasted by requiring subjects to attend to only one modality (auditory or visual), or to both, within a block of trials. Also, the light and tone stimuli were randomly 
presented at one of two spatial locations to the left and right of a central fixation point. Combination of the two modalities and two locations provided a total of four possible information sources (left-light, left-tone, right-light, right-tone). In the focusedattention condition, trials were blocked by modality, essentially halving the uncertainty, and leaving a stimulus set size of two (set size here refers to the number of distinct sources of information among which attention must be divided). The subject's task in this condition was a two-alternative (left or right) forced-choice judgment. In the divided-attention condition, auditory and visual trials were mixed randomly within a block. Subjects were required to divide their attention among the four potential sources (set size $=4$ ) in this four-alternative forced-choice task. Following Fidell's (1970) terminology, we will refer to the focused and divided-attention conditions, respectively, as homomodal and heteromodal blocks.

\section{THEORETICAL FRAMEWORK}

The set-size problem can be stated more formally if we conceptualize the sensory event or internal representation elicited by a source of information (signal or noise) as a random variable whose parameters vary with the quality of the information obtained. The question then becomes: Does increasing the number of sources requiring attention affect the parameters of the random variables or only those of the decision processes? To answer this question, we can formulate several predictions of the effects on performance of changes in set size. These predictions will differ as a function of the assumptions made about the parameters of the internal random variables and about the role of attention.

Let the random variable $\mathbf{X}_{\mathbf{k i}}$ be the internal representation of source $\mathbf{k}$ when stimulus $i$ is presented, where $i$ has the value zero on noise trials and one on signal trials. It is assumed that the source chosen is the one evoking the strongest impression of the presence of a signal, that is, the largest value of $\mathbf{X}_{\mathbf{k i}}$. Let us also assume, initially, that increasing the number of attended sources from two to four does not change the parameters of the internal random variable corresponding to each source. That is, assume, for now, no attentional effect. Then, for a given probability distribution on the random variable $\mathbf{X}_{\mathbf{k}}$, it is possible to calculate the expected change in the probability of a correct source judgment as set size increases from two to four.

The upper two curves in Figure 1 were generated in this way for assumed Gaussian and exponential probability distributions. In this figure, the predicted probability of a correct source choice in the heteromodal condition $\left(\mathrm{P}_{4}\right)$ is plotted against the corresponding probability for the homomodal condition $\left(\mathrm{P}_{2}\right)$. The

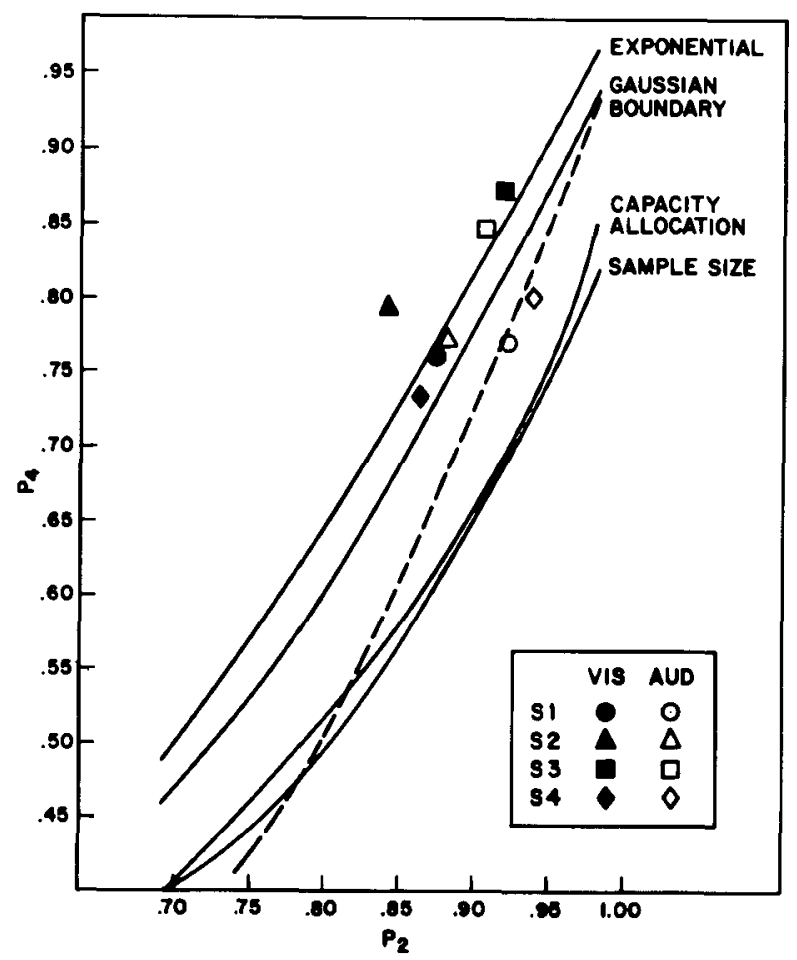

Figure 1. The relation between $P_{2}$ (probability of a correct source choice in the homodal task) and $P_{4}$ (probability of a correct source choice In the heteromodal task) predicted by several models, and the data obtained from four subjects.

subscripts refer to set size in the respective conditions. These two functions have in common assumptions of normally distributed random variables, equal variances for signal and noise distributions, and no effect of the number of to-be-attended-to sources on the parameters of these distributions. Note that the curve based on the exponential distribution predicts the smallest performance decrement as set size increases from two to four.

The two curves in Figure 1 labeled "capacity allocation" and "sample size" predict substantially larger drops in accuracy on the four-alternative task. These two curves differ from the Gaussian and exponential functions principally in that the capacity allocation and sample size models both rest on the assumption that dividing attention does affect the parameters of the internal random variables, that is, that there is an attentional effect. There are, of course, other differences between the models, less pertinent to the present discussion. For a more detailed description of these models, see Shaw (1980) and Shaw and Shaw (1977).

One strategy for discriminating attentional from decision-making effects would be to compare the fit of data from this type of set-size experiment to each of the four models we have presented. However, there may exist models based on other probability 
distributions, which predict greater drops in $\mathbf{P}_{4}$ relative to $P_{2}$, without assuming any attentional effect. This possibility makes it difficult to claim that a performance decrement observed in an empirically generated function lying significantly below the Gaussian curve in Figure 1 must be due to an attentional effect. Another approach to this problem is to pose the following question: Is there an upper bound on the drop in accuracy that could be predicted by a model assuming no effect of dividing attention on the quality of the internal stimulus representations? An answer to this question has been proposed in Shaw (1980) and is illustrated by the "boundary" function in Figure 1. If it can be assumed that subjects divide their attention among the sources on each trial (i.e., that none of the sources is completely ignored), then we can claim that, for any empirical function lying significantly below and to the right of the boundary curve, an attentional effect is strongly indicated.

Derivation of the boundary function has been presented in detail previously (Shaw, 1980). The model is nonparametric with regard to the underlying probability distributions. It assumes only that the signal source chosen is the one evoking the strongest impression of the signal's presence and, of course, that increasing set size has no effect on the means and variances of the random variables. In simplified form, the model for the boundary function may be stated as

$$
\mathbf{P}_{\mathbf{N}} \geqslant \mathbf{P}_{\mathbf{2}}^{\mathbf{N}-1} \text {, }
$$

where $P_{N}$ is the probability that the value of the signal random variable exceeds that of any of the $\mathrm{N}-1$ noise variables. The boundary function in Figure 1, then, is simply $\mathbf{P}_{2}^{4-1}$, or $\mathbf{P}_{2}^{3}$, and is the appropriate function for evaluating data from the present experiment.

\section{EXPERIMENT}

The experiment to be reported had two goals. One was to examine the effects of dividing attention between modalities using the framework just outlined. A second goal was to attempt to replicate a previously reported result (Mulligan \& Shaw, 1980) concerning the manner in which information is pooled across modalities in a detection task. In order to achieve both goals, subjects were required to make two responses on each trial-a "yes-no" detection response and the forced-choice judgment described earlier. Only data from the heteromodal condition were used to investigate the second problem. Analysis of the detection data from these blocks compared performance on unimodal trials (those on which a signal was presented in one modality) with that on bimodal trials (those on which concurrent light and tone signals were presented at the same spatial location). As was the case in the earlier study, pooling of information across modalities was best described by a version of the independent decisions model in which attention is shared equally between modalities on each trial. This model, its predictions, and the relevant data from the current experiment are presented in the Appendix.

The detection data have no direct implications for the primary focus of the present paper-the problem of dividing attention between modalities. The two problems are related, however, and have been discussed in greater detail elsewhere (Shaw, 1980, in press). At this point, we want only to point out that the yes-no task and some of the trial types described in the procedure below (bimodal trials and trials on which a light or tone is presented at both locations) are not relevant to the problem addressed in this paper.

\section{Method}

Subjects. Four Rutgers University undergraduates served as subjects. All had normal or corrected-to-normal visual acuity, and auditory sensitivity for $.125-8.0 \mathrm{~Hz}$ within $10 \mathrm{~dB}$ of audiometric zero in each ear. The subjects were paid $\$ 3 / \mathrm{h}$.

Apparatus. Visual and auditory signal and noise stimuli were generated using equipment similar to that described in Mulligan and Shaw (1980). The apparatus included standard electroacoustical components for generating and shaping auditory stimuli, the electronics from two Scientific Prototype 800F two-channel tachistoscopes for generating and timing visual stimuli, and a series of timers for controlling the sequence of trial events.

The subject's sound-attenuated room contained two bimodal stimulus arrays, each comprising a KLH Model 12.5 loudspeaker, over which auditory signals embedded in white noise were presented, and two tachistoscope lamps, one providing background "noise" illumination and the other delivering signal light pulses. Light stimuli were visible through an $8 \times 10 \mathrm{~cm}$ opening at the front of the otherwise light-tight lamp enclosure. A piece of white Plexiglas covered the opening, creating an area of uniform luminance that subtended a visual angle of $2.8 \mathrm{deg}$ at the position of the observer, $2 \mathrm{~m}$ away.

With the lamp enclosures placed on top of the speakers, the two bimodal stimulus arrays were mounted on shelves projecting from adjacent corners of the room. A $28 \times 34 \mathrm{~cm}$ piece of black plastic foam speaker grill $(2.5 \mathrm{~cm}$ thick) covered the front surface of the speaker and lamp enclosure, giving the illuminated area a grainy, textured appearance. A small red warning-fixation light was mounted on the wall of the booth between the two stimulus arrays and directly opposite the observer's chair. With the observer orienting toward and fixating on this lamp, the left and right stimulus arrays were located, respectively, at 325 and 35 deg azimuth along the horizontal meridian.

Audio white noise was maintained for all subjects at $55 \mathrm{dBA}$ measured at the position of the observer's head (Bruel \& Kjaer, Type 2203 sound-level meter). The background luminance level, measured at the surface of the speaker grill, was $.40 \mu \mathrm{W} / \mathrm{cm}^{2}$ (Tektronix J-16 photometer). Measured intensity of signal plus noise for the four subjects ranged from 55 to $57 \mathrm{dBA}$ for the audio stimulus, and from .42 to $.47 \mu \mathrm{W} / \mathrm{cm}^{2}$ for the light stimulus. The stimulus intensities were monitored daily to assure reliability.

Procedure. The temporal sequence for a single experimental trial is illustrated in Figure 2. A 1.0-sec pulse of the warning/ 


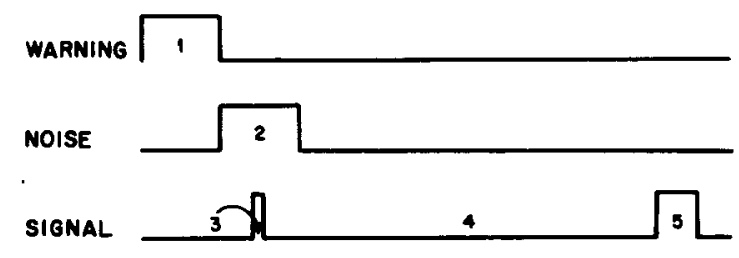

SECOND

Figure 2. Sequence of trial events: (1) 1.0-sec warning/fixation Interval, (2) 1.0-sec noise Interval, (3) 100-msec signal Interval, (4) 4.5-sec reaponse interval, (5) 500 -msec feedback interval.

fixation light marked the beginning of each trial. This was followed by a 1.0 -sec period during which auditory and visual noise were presented at both stimulus locations. On $60 \%$ of the trials, a signal (light, tone, or both), $100 \mathrm{msec}$ in duration, was embedded in the noise, beginning 500 msec after its onset. Noise alone was presented on the remaining $40 \%$ of the trials. Offset of the noise stimuli marked the beginning of a 4.5-sec interval during which subjects made their responses. This was followed by a 500 -msec period during which the signal for that trial was repeated in the absence of noise. This interval was blank, of course, on noiseonly trials. The subjects were provided with a two-position switch on the chair arm with which they could stop and restart the trial sequence, and thereby control the pace of the experiment. This switch was used very rarely, however, the subjects generally preferring the trials to cycle without interruption.

Blocks of 100 trials were administered either homomodally or heteromodally. Homomodal blocks (focused attention with respect to modality) consisted of all auditory or all visual signals. Within each block of homomodal trials, signals could be presented via the left or the right stimulus array, or at both locations simultaneously. Each of these three trial types occurred with an equal probability. Order of presentation of trials within a block was random, with the constraint that each homomodal block contained the same number of trials of each type.

Heteromodal blocks (divided attention) contained both unimodal (auditory and visual) trials and bimodal trials, on which both light and tone signals were presented simultaneously. These three signal types were equiprobable within a heteromodal block. Half of the trials of each type were delivered via the left stimulus array and half via the right array. There were no trials in the heteromodal condition on which signals occurred in both locations.

On each trial, in both homomodal and heteromodal conditions, the subjects were asked to make both a detection response and a forced-choice location response (left or right). They were required to make the second judgment regardless of their detection response. In the homomodal condition, instructions for the forcedchoice response required subjects to attend equally and simultaneously to both stimulus arrays and to choose the location that gave the strongest impression of a signal's presence. This instruction provided a decision rule for choosing a location on trials when the subject's detection criteria were exceeded at both locations or at neither location.

With heteromodal presentation, the subjects were asked, in addition to the detection and location responses, to make a forcedchoice modality judgment (light or tone). They were instructed to divide their attention equally between the possible signal sources on each trial and to choose the combination of location and modality giving the strongest impression of the signal's occurrence. For the purposes of our analysis, the two forced-choice responses are more conveniently viewed as a single four-alternative forced-choice task in which the subject chooses one of four possible location/modality combinations.

After screening for normal vision and hearing, each subject served in eight practice sessions comprising three or four blocks of 100 trials. During practice, each subject completed at least 12 homomodal blocks (6 visual and 6 auditory) and 12 heteromodal blocks. During the first five practice sessions, stimulus intensity was gradually reduced until performance reached the desired level. Data were collected in eight subsequent sessions, each consisting of 20 warm-up trials followed by three blocks of 100 trials. Homomodal and heteromodal conditions were presented alternately on successive days of the experiment.

\section{Results}

Data from the experiment are presented along with the five theoretical set-size functions in Figure 1. For each subject, $P_{4}$, the probability of correctly identifying both the location and the modality of a signal in the heteromodal task, is plotted as a function of $P_{2}$, the probability of a correct location judgment in the homomodal conditions. Since there were small, but significant, differences in accuracy between modalities for each of the subjects, auditory and visual data were plotted separately. Standard deviations for the data points ranged from .01 to .03 . Six of the eight points lie above the boundary curve. Three of these are significantly different from the boundary $(p<.05)$. The remaining five points do not differ from the prediction of the boundary function.

\section{DISCUSSION}

The results of this experiment provide no evidence for the existence of an attentional effect when attention must be divided between simple light and tone signals occurring at one of two positions in space. The data from all four subjects were either above or not significantly different from the boundary curve. The observed differences in accuracy between focused (homomodal) and divided (heteromodal) attention conditions can be attributed entirely to nonattentional factors.

Although the paradigms differ considerably, our conclusions are similar to those reached by Eijkman and Vendrik (1965) and Shiffrin and Grantham (1974); namely, there is no evidence that dividing attention between modalities adversely affects the quality of the information obtained by each. For several reasons, we feel that our results permit a stronger statement of this conclusion. In both of these previous studies, the conclusions are based on a hypothesis of no effect. In our investigation, the question is not whether there is an effect, but rather, what the magnitude of the effect is. Also, in both of the earlier studies, the conclusions are based, at least in part, on analysis of $d^{\prime}$ measures, requiring the authors to assume that signal and noise were normally distributed and that subjects' criteria were 
similar across conditions. Our analysis requires no assumptions about these parameters.

Our findings can be contrasted to those of Massaro and his co-workers from three experiments in which they observed significant divided-attention decrements. In the experiment most relevant to this discussion (Massaro \& Warner, 1977, Experiment 2), auditory and visual stimuli were presented either sequentially or simultaneously on a given trial. In this paradigm, the opportunity for error in the focused (sequential) and divided (simultaneous) conditions is equated, since the same number of stimuli are presented in each case. Therefore, any drop in accuracy in the simultaneous condition must be due to some other factor, most likely to the possibility that less attention is available to process each stimulus. As Massaro and Warner point out, the attention effect they observed may be reconciled with the nonattentional results from other studies if the nature of the stimuli and the task demands is taken into account. For their visual and auditory tasks, Massaro and Warner required subjects to recognize a letter (" $U$ " or "V") and a tone (high, $880 \mathrm{~Hz}$, or low, $800 \mathrm{~Hz}$ ). In the other studies we have discussed, the present experiment included, subjects were required only to detect a change in the energy level of a stimulus.

Perhaps tasks requiring only simple energy detection are not capacity limited, while those requiring further processing-recognition, for example-will show set size effects. Judging from the literature on selective attention with purely visual displays, however, it appears certain that this statement is an oversimplification. Schneider and Shiffrin's (1977) distinction between "automatic" (not influenced by capacity limitations) and "controlled" (subject to capacity-limitation effects) processing provides a framework for illustrating this point. Set-size effects attributable to attentional variables have been demonstrated in several experiments (e.g., Shaw \& Mulligan, Note 2) in which subjects were required to discriminate target characters from distractors-a task requiring some degree of pattern recognition. In experiments using well-practiced, easily discriminable stimuli, however, Schneider and Shiffrin have shown that, under certain circumstances, patterned stimuli can receive automatic processing. Increasing set size in tasks requiring discrimination of these "consistently mapped" stimuli does not produce a performance decrement attributable to divided attention.

There is also some evidence that processing is not necessarily automatic, and free of capacity limitations, when simple "energy detection" stimuli are used. Posner and his colleagues (Posner, 1980; Posner, Nissen, \& Ogden, 1978) have presented data from several experiments which indicate, contrary to what might be expected from the results of the present study, that performance decrements are obtained when attention is divided between the locations at which a light flash can occur. In a typical experiment, Posner et al. present a light flash to the right or left of a central fixation point, and record either simple or forced-choice reaction times (RTs). The RTs are compared across conditions which differ with respect to the precued probability of a target at each location. In general, RT to a stimulus flash at some location is found to decrease with increasing a priori stimulus probability for that location. While it might be concluded from this result that subjects are allocating more of their fixed attentional resource to the high-probability locations, there is an alternative explanation. The differences in RT could also be accounted for if subjects shifted their decision criteria to correspond to the cued stimulus probabilities at each location. An experiment with simple light-flash stimuli, using the paradigm described in the present paper, might help to clarify this issue.

The results of a recent study by Kramer, Yager, Graham, and Shaw (1980), examining the effects of set size on recognition of spatial frequencies, have some bearing on this problem. Their analysis (identical to that used in this paper) revealed no attentional effect on identification of spatial frequencies as set size was increased from one to four. Their data fell between the predictions of the Gaussian and exponential decision models illustrated in Figure 1. If the identification of spatial frequencies is viewed as a rudimentary process in visual information processing, a precursor to later pattern recognition stages, then Kramer et al.'s results are inconsistent with the attentional explanation of Posner's results. The results favor, instead, an interpretation in which both spatial frequency analysis and simple energy detection are seen as automatic processes in which attention plays no differential role.

The relationship between stimulus complexity and set size, both within and between modalities, should be investigated in greater detail in future experiments. Techniques that address the locus-of-capacitylimitation problem, such as those used by Duncan (1980) and Hoffman (1979) might be fruitfully applied to this investigation.

It is important to point out that the conclusions drawn from the data in the present experiment, and from others in which data are compared with a similarly derived boundary function, are dependent on having identified the number of attended sources. It is possible to conceive of conditions under which the number of sources of information to which a subject is attending is not equal to the number of sources we have specified. ${ }^{1}$ It might be argued, for example, that extraneous distractors or input from memory constitute additional sources of information. If this were the case, our assumed number of 
sources (two vs. four) would underestimate the true number. Fortunately, such an underestimation of the number of to-be-attended sources will not influence the group of theoretical curves in Figure 1, if it can be assumed that the extraneous sources are not treated as response alternatives.

We mentioned earlier the importance of the assumption that no source is completely ignored. Suppose, for example, that our subjects had chosen to ignore the information presented at one location, and that our data were actually based on one vs. three sources instead of two vs. four as we had assumed. In this case, the two vs. four boundary curve would overestimate true boundary performance, thus increasing the likelihood of incorrectly concluding that an attention effect existed. Another sense in which we may have overestimated the number of to-beattended sources is suggested by Kubovy's (1981) theory of "indispensable attributes." According to this theory, the spatial location of a stimulus is an indispensable attribute for visual stimuli, but not for auditory stimuli. Thus, in our auditory homomodal condition, attending to two spatial positions would not demand resources in the same way that dividing attention between spatial positions does in the visual homomodal condition. From this point of view, attention is divided only three ways in our heteromodal condition: visual-left, visual-right, and auditory. If this interpretation is correct, then the theoretical curves in Figure 1 are inappropriate, since our experiment actually involves a comparison of set size one vs. three (auditory homomodal vs. heteromodal) or two vs. three (visual homomodal vs. heteromodal). Kubovy suggests that the pitch dimension in audition is analogous to the spatial dimension in vision. In future experiments investigating attention divided among multiple auditory signals, then, it would be of interest to use signals differing in frequency rather than in spatial location or ear of presentation.

\section{REFERENCE NOTES}

1. Shaw, M. L. Attending to multiple sources of information: II. The effects of selectivity and uncertainty on performance. Manuscript in preparation.

2. Shaw, M. L., \& Mulligan, R. M. Attention and set size effects in visual search. Manuscript submitted for publication.

3. Swensson, R. G., \& Judy, P. F. Detection and location of noisy visual targets. Paper presented at the $21 \mathrm{st}$ annual meeting of the Psychonomic Society, St. Louis, November 1980.

\section{REFERENCES}

Duncan, J. The locus of interference in the perception of simultaneous stimuli. Psychological Review, 1980, 87, 272-300.

EGeth, H. Attention and preattention. In G. H. Bower (Ed.), The psychology of learning and motivation (Vol. 7). New York: Academic Press, 1977.

Eijkman, E., \& Vendrik, A. J. Can a sensory system be specified by its internal noise? Journal of the Acoustical Society of America, 1965, 37, 1102-1109.
Erikgen, C. W., \& Spencer, T. Rate of information processing in visual perception: Some results and methodological considerations. Perception \& Psychophysics, 1972, 11, 169-171.

Fidell, S. Sensory function in multimodal signal detection. Journal of the Acoustical Society of America, 1970, 47, 1009 1015.

GARDNER, G. T. Evidence for independent parallel channels in tachistoscopic perception. Cognitive Psychology, 1973, 4, 130 155.

Graham, N. Visual detection of aperiodic spatial stimuli by probability summation among narrowband channels. Vision Research, 1977. 17, 637-652.

Hoffman, J. E. A two-stage model of visual search. Perception \& Psychophysics, 1979, 25, 319-327.

KinchlA, R. A. Temporal and channel uncertainty in detection: A multiple observation analysis. Perception \& Psychophysics, $1969,5,129-136$.

Kramer, P., Yager, D., Graham, N., \& Shaw, M. L. Recognition of spatial frequency: Effect of set size. Investigative Ophthalmology and Visual Science, April 1981. (Suppl.)

KuBovy, M. Concurrent pitch-segregation and the theory of indispensable attributes. In M. Kubovy \& J. Pomerantz (Eds.), Perceptual organization. Hillsdale, N.J: Erlbaum, 1981.

Massaro, D. W., \& KaHN, B. J. Effects of central processing on auditory recognition. Journal of Experimental Psychology, $1973,97,51-58$.

Massaro, D. W., \& Warner, D. S. Dividing attention between auditory and visual perception. Perception \& Psychophysics, 1977, 21, 569-574.

Mulligan, R. M., \& Shaw, M. L. Multimodal signal detection: Independent decisions vs. integration. Perception \& Psychophysics, 1980, 28, $471-478$.

Posner, M. I. Orienting of attention. Quarterly Journal of Experimental Psychology, 1980, 32, 3-25.

Posner, M. I., Nissen, M. J., \& Ogden, W. C. Attended and unattended processing modes: The role of set for spatial attention. In H. L. Pick \& I. J. Saltzman (Eds.), Modes of perceiving and processing information. Hillsdale, N.J: Erlbaum, 1978.

Schneider, W., \& Shiffrin, R. M. Controlled and automatic human information processing: I. Detection, search and attention. Psychological Review, 1977, 84, 1-66.

SHAw, M. L. Identifying attentional and decision-making components in information processing. In $R$. S. Nickerson (Ed.), Attention and performance VIII. Hillsdale, N.J: Erlbaum, 1980.

Shaw, M. L. Attending to multiple sources of information: I. Integrating sources of information. Cognitive Psychology, in press.

Shaw, M., \& SHAw, P. Optimal allocation of cognitive resources to spatial locations. Journal of Experimental Psychology: Human Perception and Performance, 1977, 3, 201-211.

Shiffain, R. M., \& Gardner, G. T. Visual processing capacity and attentional control. Journal of Experimental Psychology, $1972,93,72-83$.

Shiffrin, R. M., \& Grantham, D. W. Can attention be allocated to sensory modalities? Perception \& Psychophysics, 1974, 15, 460-474.

Sperling, G., \& Melchner, M. J. The attention operating characteristic: Examples from visual search. Science, 1978, 202, 315-318.

\section{NOTE}

1. We are indebted to an anonymous reviewer for bringing some of these conditions to our attention.

\section{APPENDIX}

Given a detection task in which subjects are required to make a binary response based on multiple stimulus sources, 
how might the information about individual sources be combined in formulating a detection response? One class of response selection rules which has accounted for a good bit of data from this type of experiment is characterized by the independent decisions model. The model has also been called "probability summation" by Graham (1977) in the spatial frequency literature and the "extreme detector" model by Swensson and Judy (Note 3) in work on target detection in radiographs. According to the independent decisions model, an observer makes a separate yes-no decision about each source of information, and the results of these independent judgments are then combined to obtain a response. The response is positive whenever the number of positive decisions regarding individual sources exceeds some criterion value.

The independent decisions model and its predictions will be described below in terms of the paradigm of the present experiment. For this analysis, we are concerned only with performance in the heteromodal condition. On each heteromodal trial, one of the possible stimuli, $S_{i j k l}$, was presented. The four subscripts, i, j, k, and l, refer to the four possible stimulus sources: left-light, left-tone, rightlight, and right-tone, respectively. Each subscript can assume one of two values-" 1 ," if a signal is embedded in the noise presented at that source, or " 0 ," if only noise is presented. In this experiment the stimulus set was limited to the following: $S_{0000}, S_{1000}, S_{0100}, S_{0010}, S_{0001}, S_{1100}, S_{0011}$.

Performance is expressed in terms of $P_{\mathrm{ijk}}$, the conditional probability of a "no" response given stimulus $S_{i j k l}$. For example, $P_{0100}$ is the probability of a "no" response given that a tone was presented at the left stimulus array and noise only at the other three sources.

Within this model, the sensory response evoked by each stimulus is represented as a random variable, $X_{m p}$, where $\mathrm{m}$ denotes one of the four signal sources ( 1 for left-light, 2 for left-tone, 3 for right-light, and 4 for right-tone), and $p$ takes value " 0 " on noise-only trials and " 1 " on signalplus-noise trials. Subjects in the experiment were instructed to respond "yes" if they detected a signal at any of the four sources. In terms of the independent decisions model, then, the decision rule is to respond "yes" if the value of any of the $X_{m p}$ exceeds its corresponding criterion, $\beta_{m}$.

In a previous paper, Shaw (in press) developed a set of parameter-free predictions for three alternative versions of the independent-decisions model. These versions differ in their assumptions about how attention is allocated to sources on each trial. Only the sharing model will be presented here. More detailed descriptions of all three versions, and their predictions, have been presented previously (Shaw, 1980, in press). According to the sharing model, on each trial of a detection experiment, a subject divides attention among the relevant sources of information. While capacity is not necessarily apportioned in equal amounts to each source, it is assumed that the proportion assigned to each source and the various decision criteria, $\beta_{\mathrm{m}}$, remain constant across trials. It follows, then, that the conditional probability of a "no" response given the stimulus $\mathrm{S}_{\mathrm{ijk}}$, is the product of the probabilities that the random variable representing each source does not exceed its corresponding criterion:

$$
P_{i j k l}=P\left(X_{1 p}<\beta_{1}\right) P\left(X_{2 p}<\beta_{2}\right) P\left(X_{3 p}<\beta_{3}\right) P\left(X_{4 p}<\beta_{4}\right) .
$$

For example, the "no" probability for trials on which a tone is presented via the left stimulus array is:

$$
P_{0100}=P\left(X_{10}<\beta_{1}\right) P\left(X_{21}<\beta_{2}\right) P\left(X_{30}<\beta_{3}\right) P\left(X_{40}<\beta_{4}\right) .
$$

After thus obtaining the $P_{i j k \mid}$ s for the seven different stimuli used in the experiment, the following equal products property for "no" responses can be derived:

$$
\left.\mathbf{P}_{1000} \mathbf{P}_{0110} \mathbf{P}_{0010} \mathbf{P}_{0001}=\mathbf{P}_{1100} \mathbf{P}_{0011}^{\prime} \mathbf{2} \mathbf{P}_{0000}\right) \text {. }
$$

Furthermore, after logarithmic transformation, it can be shown that:

$$
\begin{aligned}
\ln P_{1000}+\ln P_{0100}+\ln P_{0010} & +\ln P_{0001} \\
& =\ln P_{1100}+\ln P_{0011}+2\left(\ln P_{0000}\right) .
\end{aligned}
$$

In other words, the sharing model predicts additivity of "no" probabilities under the logarithmic transformation.

This prediction, along with those of two other versions of the independent decisions model and a Gaussian integration model, was compared with data obtained in the heteromodal condition. The sharing model was evaluated using a $z$ statistic that tests the extent to which the log transformed data deviate from the additivity prediction. The model predicts that $\Delta=\ln P_{1100}+\ln P_{0011}+2\left(\ln P_{0000}\right)$ $-\left(\ln P_{1000}+\ln P_{0100}+\ln P_{0010}+\ln P_{0001}\right)=0$. For a large sample, the sampling distribution of the estimate of $\ln P_{\mathrm{ijk}} \mathbf{l}$, In $\hat{P}_{i j k l}$, can be approximated by a normal distribution with mean $\ln P_{i j k l}$ and variance $\left(1-P_{i j k l}\right) / N_{i j k l} P_{i j k l}$. Therefore, the appropriate test of the null hypothesis $(\Delta=0)$ is:

\begin{tabular}{|c|c|c|c|c|c|c|c|c|c|c|}
\hline \multirow[b]{2}{*}{ Subject } & \multicolumn{7}{|c|}{ Probability of a "No" Response } & \multicolumn{3}{|c|}{$\begin{array}{l}\text { Test for Additivity of } \\
\text { Transformed Data (z Score) }\end{array}$} \\
\hline & $P_{0000}$ & $P_{1000}$ & $P_{0100}$ & $P_{0010}$ & $P_{0001}$ & $P_{1100}$ & $P_{0011}$ & Sharing & Mixture 1 & Integration \\
\hline $\begin{array}{l}1 \\
2 \\
3 \\
4\end{array}$ & $\begin{array}{l}.84 \\
.88 \\
.95 \\
.91\end{array}$ & $\begin{array}{l}.31 \\
.33 \\
.28 \\
.38\end{array}$ & $\begin{array}{l}.24 \\
.29 \\
.24 \\
.28\end{array}$ & $\begin{array}{l}.28 \\
.41 \\
.23 \\
.37\end{array}$ & $\begin{array}{l}.33 \\
.39 \\
.31 \\
.29\end{array}$ & $\begin{array}{l}.08 \\
.13 \\
.09 \\
.07\end{array}$ & $\begin{array}{l}.09 \\
.18 \\
.11 \\
.06\end{array}$ & $\begin{array}{r}.59 \\
.33 \\
-1.26 \\
2.13^{*}\end{array}$ & $\begin{array}{l}-7.18^{* *} \\
-5.27^{* *} \\
-11.38 \dagger \\
-6.64^{* *}\end{array}$ & $\begin{array}{l}4.05^{* *} \\
4.52^{* *} \\
8.46 \dagger \\
3.73^{* *}\end{array}$ \\
\hline
\end{tabular}

$$
z=\frac{\Delta}{S_{\Delta}},
$$

where $S_{\Delta}$ is the estimated standard deviation of $\Delta$.

Table 1

Detection Data From the Heteromodal Condition

$* p<.05 . \quad * * p<.01 . \quad$ tp<.001. 
The data from four subjects are reported in Table 1. A significant $\mathrm{z}$ score represents deviation from additivity and, thus, constitutes evidence against the sharing model. Data from three subjects supported the sharing model and rejected the other models tested. The fourth subject's data were consistent with an alternative version of the independent decisions model, in which it is assumed that the observer allocates attention to potential sources on each trial in a probabilistic fashion and that the criterion associated with each source varies from trial to trial with changes in the allocation of attention. This model, labeled the "type 2 mixture model" by Shaw (1980), is similar to Sperling and Melchner's (1978) "attention switching model."
The results closely replicate those of our previous multimodal detection study (Mulligan \& Shaw, 1980). Combining the data from these two similar studies, we see that the performance of all eight subjects was in accord with the predictions of the independent decisions model. Data from six subjects favored the sharing version, while the other two subjects performed according to the Type 2 mixture model.
(Manuscript received May 4, 1981; revision accepted for publication August 10, 1981.) 